\title{
Adsorption and photoreactivity of CdSe nanoparticles at liquid|liquid interfaces
}

\author{
Bin Su, David J. Fermín ${ }^{1}$, Jean-Pierre Abid, Nicolas Eugster, Hubert H. Girault * \\ Laboratoire delectrochimie Physique et Analytique, Institut des Sciences et Ingénierie Chimiques, Ecole Polytechnique Fédérale de Lausanne, \\ CH-1015 Lausanne, Switzerland
}

Received 20 December 2004; received in revised form 28 January 2005; accepted 28 January 2005

Available online 26 July 2005

\begin{abstract}
The voltage induced assembly and photoreactivity of cadmium selenide $(\mathrm{CdSe})$ nanoparticles protected by mercaptosuccinic acid are studied at the polarisable interface between water and 1,2-dichloroethane electrolyte solutions. Cyclic voltammograms and admittance measurements show an increase of the interface excess charge associated with the adsorption of CdSe nanoparticles as the Galvani potential difference is tuned to negative values with respect to the potential in the organic phase. Within the potential range where the nanoparticles are adsorbed, band-gap illumination leads to heterogeneous electron transfer from CdSe nanoparticles to electron acceptors located in the organic phase. The interfacial Galvani potential difference plays an important role in these phenomena, as it affects the interfacial density of the nanoparticles, as well as the driving force for the electron transfer. The photocurrent efficiency also strongly depends on the formal redox potential of the electron acceptor, indicating that the heterogeneous photoreaction is kinetically controlled. The interfacial electron transfer occurs via depopulation of the deep trap states in the band gap. Analysis of the photocurrent transient responses reveals that the magnitude of the instantaneous photocurrent upon illumination is determined by the kinetics of heterogeneous electron transfer, while photogenerated holes are swiftly captured by species present in the aqueous phase. The photocurrent decay upon constant illumination is associated with the diffusion of the acceptor to the interfacial region. From the phenomenological point of view, the photoelectrochemical behaviour of CdSe nanoparticles can be compared to a self-assembled ultrathin p-type semiconductor photoelectrode.
\end{abstract}

(C) 2005 Elsevier B.V. All rights reserved.

Keywords: Adsorption; Photocurrent; CdSe nanoparticles; Liquid|liquid interface

\section{Introduction}

Photoinduced heterogeneous electron transfer reaction at the polarisable liquid|liquid interface has proved to be a valuable approach to artificial photosynthetic and photocatalytic processes at two phase boundaries [1-3]. At this molecular junction, photocurrent responses originating from the heterogeneous quenching of photoexcited water-soluble dyes by hydrophobic re-

\footnotetext{
* Corresponding author. Tel.: +41 21693 3151; fax: +41 21693 3667.

E-mail address: hubert.girault@epfl.ch (H.H. Girault).

${ }^{1}$ Present address: Departement für Chemie und Biochemie, University of Berne, Freiestrasse 3, CH-3012 Berne, Switzerland.
}

dox species have been studied in detail [4-11]. It has been observed that the Galvani potential difference across the liquid|liquid interface can affect the surface coverage of the photoactive species as well as the dynamics of photoinduced electron transfer and back electron transfer. Water-soluble porphyrins, chlorines and phthalocyanines have been employed as photoactive dyes at the interface between water and 1,2dichloroethane (DCE) $[4,6-10,12]$.

Recently, it has been demonstrated that titanium dioxide $\left(\mathrm{TiO}_{2}\right)$ nanoparticles can be assembled at the polarised interface between water and DCE electrolyte solutions upon applying a potential bias $[13,14]$. Bandgap illumination of the interface led to the interfacial 
transfer of holes or electrons to redox species located in the organic phase. The charge transfer reactions can be monitored by photocurrent measurements phenomenologically similar to those observed on solid photoelectrodes [15-17]. In this paper, we shall extend the study to semiconductor quantum dots sensitised liquid|liquid interfaces. Semiconductor quantum dots have all three dimensions in the nanometer range and possess unique electronic and optical properties that are strongly size dependent [18-20]. They are not only interesting from a theoretical viewpoint, but also a variety of applications have been considered. For example, luminescent semiconductor quantum dots are a promising alternative to organic dyes for fluorescence applications since they are more stable against photobleaching and have narrow and size-tunable multicolour emission as well as the functional advantages $[21,22]$. Some of the most exciting applications involve new generation of optoelectronic [23-26] and photovoltaic devices [27] and exploratory biotechnology research as ultra-sensitive biological fluorescent tags [22,28-31].

In this work, we shall describe the reversible adsorption of cadmium selenide ( $\mathrm{CdSe}$ ) quantum dots at the water|DCE interface by tuning the Galvani potential difference. In the presence of electron acceptors in the organic phase, heterogeneous photocurrent responses arising from the adsorbed CdSe nanoparticles can be recorded upon illumination of the interfacial region. Analysis of the photocurrent responses as a function of the applied potential difference and the formal redox potential of the organic phase quencher suggests that the heterogeneous electron transfer takes place from occupied trap states in the CdSe nanoparticles.

\section{Experimental}

\subsection{Synthesis of CdSe nanoparticles}

Water dispersible mercaptosuccinic acids (MSA) protected $\mathrm{CdSe}$ nanoparticles were prepared using a published protocol [32], in which $\mathrm{Na}_{2} \mathrm{Se}$ was used instead of NaHSe. In a typical synthesis, $513.3 \mathrm{mg}(2.35 \mathrm{mmol})$ of $\mathrm{CdCl}_{2} \cdot 2 \mathrm{H}_{2} \mathrm{O}$ is dissolved in $125 \mathrm{~mL}$ of water, and $866.4 \mathrm{mg}(5.77 \mathrm{mmol}) \mathrm{MSA}$ is added under stirring, followed by adjusting the $\mathrm{pH}$ to 11.2 by dropwise addition of $1 \mathrm{M}$ solution of $\mathrm{NaOH}$. After bubbling the solution with $\mathrm{N}_{2}$ for $40 \mathrm{~min}$, newly prepared solution of $1.1 \mathrm{mmol} \mathrm{Na} 2 \mathrm{Se}$ in $22 \mathrm{~mL}$ water was introduced under stirring. The resulting mixture was then subjected to a reflux at $100{ }^{\circ} \mathrm{C}$ under open air condition. During the reflux, aliquots were taken out of the flask at different times to measure the absorption spectra to monitor the growth of the nanoparticles.

The transparent solution with bright-orange colour was first concentrated on a rotary evaporator, then ace- tone was added dropwise to precipitate the nanoparticles inside the solution. The resulted mixture was stirred for $30 \mathrm{~min}$, and the precipitate and supernatant were separated through centrifugation. The precipitate was washed five times with water/acetone mixture, then dried and used in photoelectrochemical measurements.

\subsection{Spectroscopic and microscopic instrumentation}

The absorption and luminescence spectra were obtained from an Ocean Optics CHEM2000-UV-Vis spectrometer and a Perkin-Elmer LS50B Luminescence Spectrometer, respectively. High resolution transmission electron microscopy (HRTEM) images were collected from a Philips CM 300 transmission electron microscope operating with an accelerating voltage of $300 \mathrm{kV}$.

\subsection{Photoelectrochemical measurements}

The electrochemical experiments were performed using a homemade four-electrode system connected to a Stanford Research System DS335 function generator. The interfacial capacitance was calculated from admittance measurements using a Stanford Research System SR830 lock-in amplifier at a frequency of $6 \mathrm{~Hz}$ and amplitude of $10 \mathrm{mV}$ rms. A three-compartment cell featuring a cross-section of $1.53 \mathrm{~cm}^{2}$ was employed in all the measurements. The reference electrodes were placed in two separate compartments featuring Luggin capillaries. The electrolyte solutions were prepared from ultrapure water from a Milli-Q system (Millipore Milli-Q.185) and 1,2-dichloroethane (DCE) (Fluka $98 \%$ for HPLC). The composition of the electrolyte solutions is indicated in Fig. 1. The organic supporting electrolyte, bis(triphenylphosphoranylidene) ammonium tetrakis-(pentafluorophenyl)borate (BTPPATPFB), was prepared as previously reported [33]. The Galvani potential difference $\left(\Delta_{\mathrm{o}}^{\mathrm{w}} \phi\right)$ was referred to as that of the minimum of the differential capacitance curves obtained in the absence of the nanoparticles.

All photocurrent measurements were carried out with the 454-nm line of an Omnichrome S43 tunable Ar-ion laser under transmission mode. Photocurrent transients were measured employing an optical shutter with an aperture time in the range of microsecond. The incident photon flux was quantitatively determined by measuring the incident light intensity with a photomultiplier (Model 70316, Thermo Oriel Instruments).

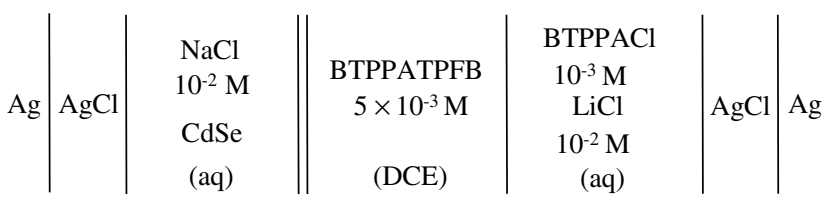

Fig. 1. The composition of the electrochemical cell. 


\section{Results and discussion}

\subsection{Characterization of CdSe nanoparticles}

Fig. 2A illustrates the temporal evolution of the absorption spectrum of the colloidal suspension of CdSe nanoparticles refluxed at $100{ }^{\circ} \mathrm{C}$. The growth of the particles is clearly evidenced by the shift of the exciton peak to longer wavelengths, which is due to the well-known quantum size effect. A typical HRTEM image of CdSe nanoparticles obtained after $120 \mathrm{~min}$ of reflux is shown in Fig. 2B. The particles tend to form agglomerates due to the strong interaction of the carboxylic groups [34], leading to a possible overestimation of the mean size and size distribution. The analysis of the micrographs showed $\mathrm{CdSe}$ nanoparticles with sizes ranging from 2 to $3.8 \mathrm{~nm}$ and resulting on an average size of $2.8 \mathrm{~nm}$. Fig. 2C shows the HRTEM image of a single CdSe crystal from the same sample. It can be observed that the particle is well crystallised with clearly resolved lattice planes. For thiol-capped CdSe clusters prepared at a low temperature, the cubic zinc blende phase is pref-
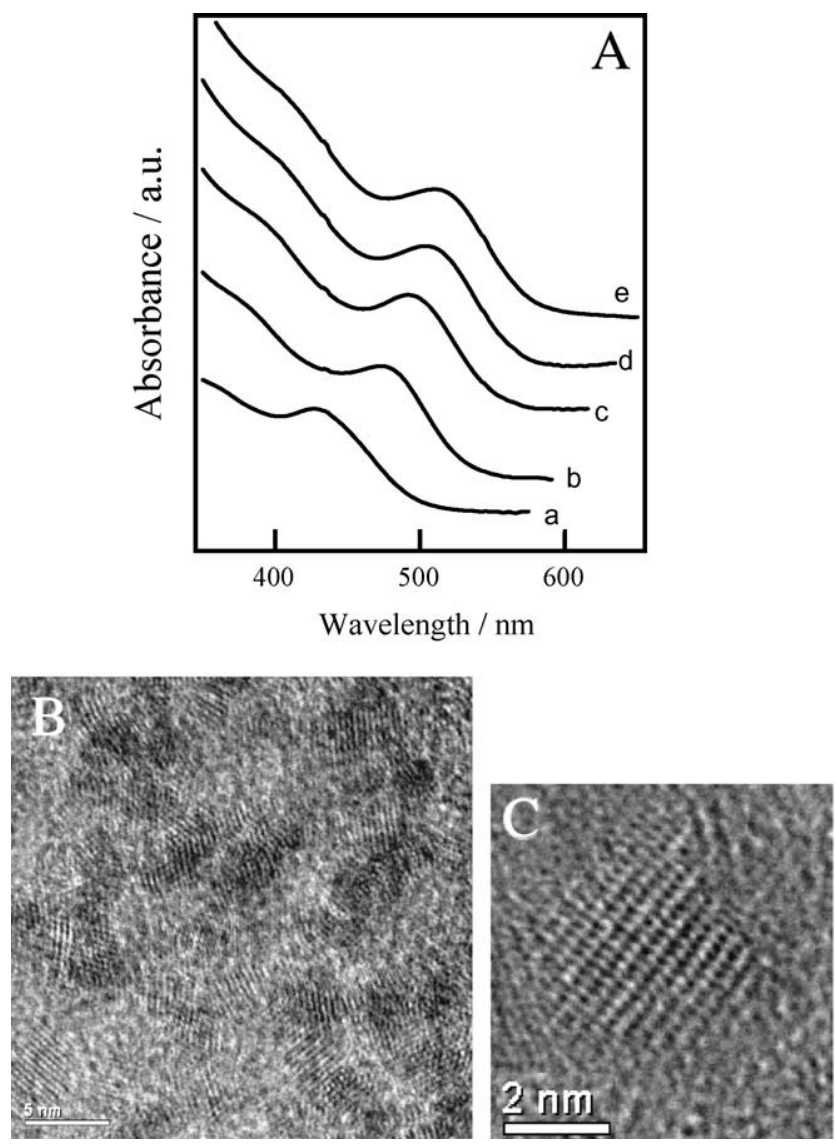

Fig. 2. (A) Temporal evolution of the absorption spectra of the reaction solution refluxed at $100^{\circ} \mathrm{C}$ : (a) $20 \mathrm{~min}$; (b) $40 \mathrm{~min}$; (c) $60 \mathrm{~min}$; (d) $80 \mathrm{~min}$; and (e) $120 \mathrm{~min}$; (B) Representative HRTEM image of CdSe nanoparticles, the scale bar is $5 \mathrm{~nm}$; (C) HRTEM image of a single CdSe nanocrystal. Images in $\mathrm{B}$ and $\mathrm{C}$ correspond to particles obtained after $120 \mathrm{~min}$ of reflux. erentially obtained [32]. Fig. 3 demonstrates the absorption and photoluminescence spectra of the CdSe colloidal suspension obtained after $120 \mathrm{~min}$ of reflux, which was used in the following photoelectrochemical measurements. The sample exhibits a broad photoluminescence band significantly red-shifted with respect to the exciton peak. Although it is expected that the size distribution contributes to the spectral broadening, the main features of the photoluminescence band reveal a distribution of energy states in the band gap acting as trap sites. The luminescence efficiency for these particles is below $0.1 \%$, indicating that the relaxation processes are dominated by "non-radiative" recombination at trap levels $[32,35]$.

The absorption cross-section per CdSe particle, $\sigma$, can be evaluated from [36]

$\sigma=4 \pi k \operatorname{Re}(\mathrm{i} \alpha)$,

where $k$ is the wave vector and $\alpha$ is the polarisability of dielectric spheres and given by

$\alpha=\frac{m_{1}^{2}-m_{2}^{2}}{m_{1}^{2}+2 m_{2}^{2}} a^{3}$,

where $m_{1}=n_{1}-\mathrm{i} k_{1}$ and $m_{2}=n_{2}-\mathrm{i} k_{2}$ are the complex refractive indices of the particle and the solvent. Using the complex refractive index of the bulk CdSe: $n_{1}=2.7$ and $k_{1}=0.7$ [37], the absorption cross-section of a $2.8 \mathrm{~nm}$ (in diameter) CdSe particle is estimated to be about $1.50 \times 10^{-15} \mathrm{~cm}^{2}$.

\subsection{Electrochemical characterisation of the adsorption of CdSe nanoparticles at the water/DCE interface}

The MSA stabilises the CdSe particles with the thiol group binding at the surface of the particle and leaving the carboxylic groups outside. As a result, the CdSe

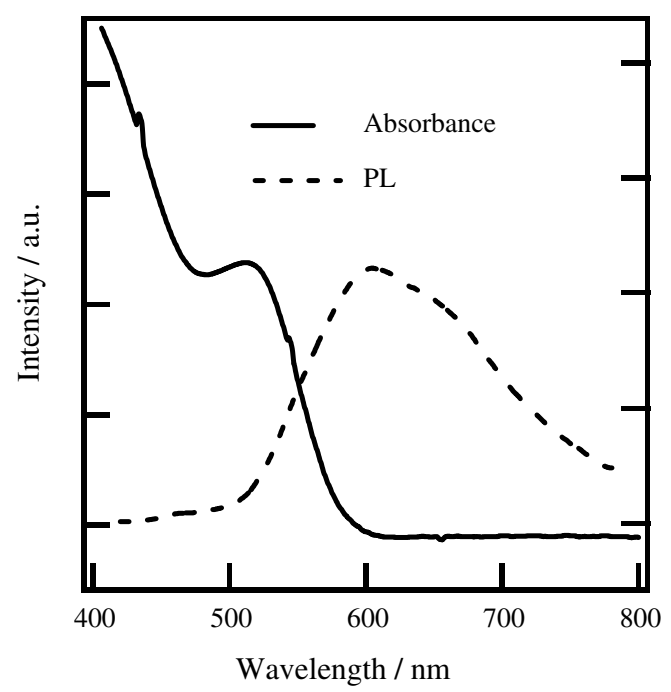

Fig. 3. Absorption and photoluminescence spectra of the CdSe colloidal suspension used in photoelectrochemical measurements. The excitation wavelength is $400 \mathrm{~nm}$. 
nanoparticles have high density of negative charges on the surface and are highly water dispersible. Cyclic voltammograms of the water|DCE interface for various concentrations of the CdSe nanoparticles in the aqueous phase are illustrated in Fig. 4A. The broad peak observed at negative Galvani potential difference is dependent on the concentration of CdSe nanoparticles and also linearly with the scan rate, suggesting that it is associated with the adsorption of CdSe nanoparticles at the interfacial boundary. This is further confirmed by differential capacitance $\left(C_{\mathrm{dl}}\right)$ measurements, as displayed in Fig. 4B. The capacitance data was extracted from admittance measurements at a frequency of $6 \mathrm{~Hz}$ and amplitude of $10 \mathrm{mV} \mathrm{rms}$, assuming that the interface can be described as a series of the uncompensated resistance and $C_{\mathrm{dl}}$. The value of $C_{\mathrm{dl}}$ can be rationalised as the
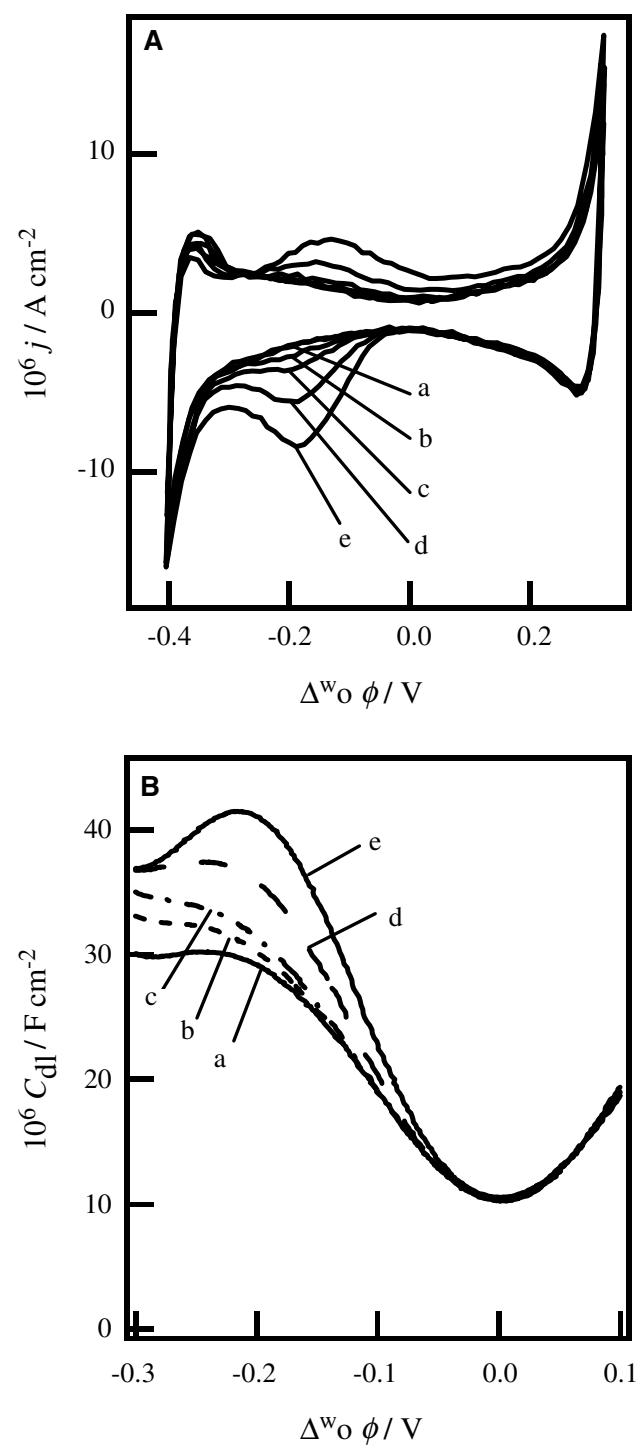

Fig. 4. Cyclic voltammograms at scan rates of $50 \mathrm{mV} \mathrm{s}^{-1}$ (A) and differential capacitance as a function of the Galvani potential difference (B) for various concentrations of the CdSe nanoparticles: (a) $0 \mathrm{~g} \mathrm{~L}^{-1}$; (b) $0.05 \mathrm{~g} \mathrm{~L}^{-1}$; (c) $0.10 \mathrm{~g} \mathrm{~L}^{-1}$; (d) $0.25 \mathrm{~g} \mathrm{~L}^{-1}$; and (e) $0.50 \mathrm{~g} \mathrm{~L}^{-1}$. derivative of the excess charge in the electric double layer with respect to the Galvani potential difference. It can be observed that the excess charge at the interfacial boundary increases at negative biases and with increasing concentration of CdSe nanoparticles. At positive potentials, the excess charge in the presence and absence of the nanoparticles is identical, indicating that the adsorption is not only voltage induced but also reversibly controlled. The same behaviour is observed upon cycling confirming that no irreversible aggregation of phase formation takes place at the liquid|liquid interface. This behaviour is comparable to that observed for gold nanoparticles stabilised by MSA at the water|DCE interface [33].

\subsection{Photoreactivity of CdSe nanoparticles at the water/DCE interface}

The redox photoreactivity of the semiconductor particles is determined by the position of the quasi-Fermi level of the electrons $\left({ }_{n} \varepsilon_{\mathrm{f}}^{*}\right)$ and holes $\left({ }_{h} \varepsilon_{\mathrm{f}}^{*}\right)$. For single crystalline materials, ${ }_{n} \varepsilon_{\mathrm{f}}^{*}$ lies very close to the conduction band edge which in the case of CdSe is associated with a redox potential $\left(E_{\mathrm{CB}}\right)$ of $-0.20 \mathrm{~V}$ vs. SHE [38]. However, $E_{\mathrm{CB}}$ can be substantially shifted to more negative potentials in nanometer sized particles. Taking the effective masses of electrons and holes as 0.11 and 0.44 , respectively [32], $E_{\mathrm{CB}}$ can be estimated as $-0.84 \mathrm{~V}$ for particles of $2.8 \mathrm{~nm}$ following the calculation reported by Burda et al. [38]. Furthermore, the band gap as calculated from the absorption spectra is $2.50 \mathrm{eV}$ [32], consequently the redox potential of holes in the valence band edge $\left(E_{\mathrm{VB}}\right)$ can be taken as $1.66 \mathrm{~V}$.

Photoinduced heterogeneous electron transfer processes involving CdSe nanoparticles and redox species in the organic phase were investigated under potentiostatic conditions. In Table 1, the formal redox potentials of various quenchers in DCE vs. the standard hydrogen electrode (SHE) are quoted as estimated by Eugster et al. $[10,11]$. In the presence of the electron acceptor $7,7^{\prime}, 8,8^{\prime}$-tetracyanoquinodimethane (TCNQ) in DCE, the photocurrent transient responses at various Galvani potential differences are illustrated in Fig. 5. The negative photocurrent indicates the transfer of a negative charge from the aqueous to the organic phase. Taking

Table 1

The formal redox potentials of various redox species in bulk DCE

\begin{tabular}{|c|c|}
\hline Redox couple & $\begin{array}{l}E^{0^{\prime}}, \mathrm{V} \\
\text { (vs. SHE) }\end{array}$ \\
\hline $7,7^{\prime}, 8,8^{\prime}$-Tetracyanoquinodimethane (TCNQ/TCNQ ${ }^{-}$) & 0.29 \\
\hline 2,3,5,6-Tetrachloro-1,4-benzoquinone (TCBQ/TCBQ $\left.{ }^{-}\right)$ & 0.17 \\
\hline 2,6-Dichloro-1,4-benzoquinone (DCBQ/DCBQ $\left.{ }^{-}\right)$ & -0.02 \\
\hline Benzoquinone $\left(\mathrm{BQ} / \mathrm{BQ}^{-}\right)$ & -0.34 \\
\hline Ferrocene $\left(\mathrm{Fc}^{+} / \mathrm{Fc}\right)$ & 0.64 \\
\hline Decamethylferrocene $\left(\mathrm{DMFc}^{+} / \mathrm{DMFc}\right)$ & 0.02 \\
\hline
\end{tabular}



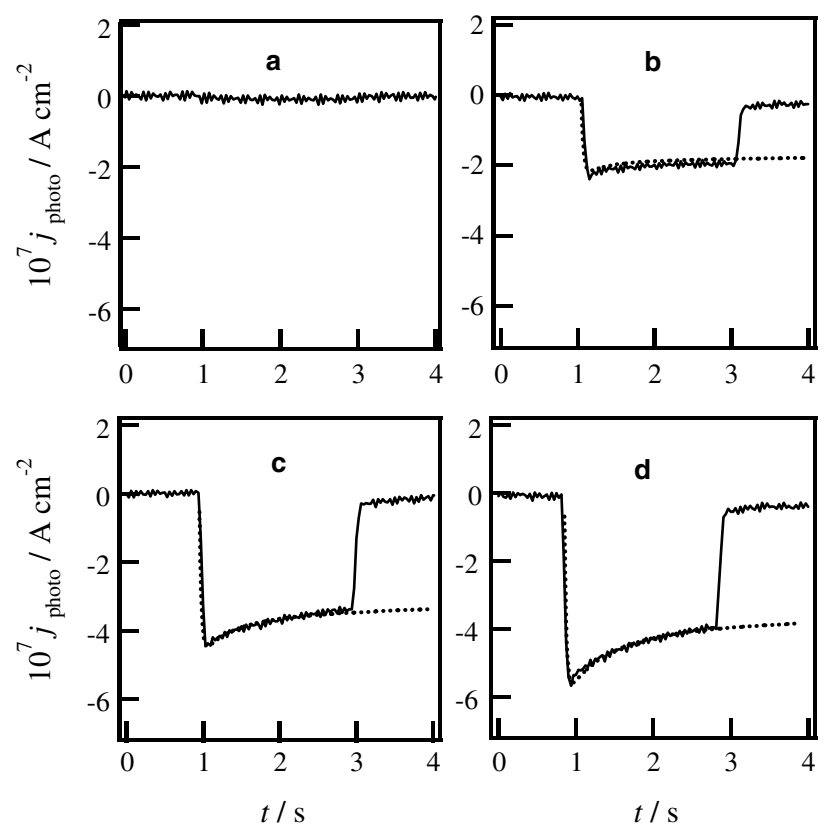

Fig. 5. Photocurrent transients arising from the photoinduced electron transfer from CdSe nanoparticles to TCNQ at various Galvani potential differences: (a) $-0.07 \mathrm{~V}$; (b) $-0.12 \mathrm{~V}$; (c) $-0.17 \mathrm{~V}$; and (d) $-0.22 \mathrm{~V}$. The dotted curves in $(\mathrm{b}-\mathrm{d})$ are simulations with the parameters $I_{0}=1.10 \times 10^{17} \mathrm{~cm}^{-2} \mathrm{~s}^{-1}, D_{\mathrm{Q}}^{\mathrm{o}}=1.05 \times 10^{-5} \mathrm{~cm}^{2} \mathrm{~s}^{-1}$ and $R C_{\mathrm{dl}}=0.025 \mathrm{~s}^{-1}$. The fits provide values of $g$ of $2.30 \times 10^{-10}$, $4.80 \times 10^{-10}$ and $6.30 \times 10^{-10} \mathrm{~mol} \mathrm{~cm}^{-2} \mathrm{~s}^{-1}$ at $-0.12,-0.17$ and $-0.22 \mathrm{~V}$, respectively. Typical values obtained for $k_{\mathrm{b}}$ was $0.40 \mathrm{~s}^{-1}$ at these three potentials while $k_{\mathrm{ps}}$ were $2.60,2.00$, and $1.25 \mathrm{~s}^{-1}$, respectively.

into account the difference in redox potential between the electrons in the conduction band edge and TCNQ, it would be expected that photoinduced electron transfer will be observed over the whole potential range. However, the photocurrent magnitude in Fig. 5 shows a strong dependence on the Galvani potential difference. This potential dependence of the photocurrent can be rationalised in terms of: (i) changes in the particle density in the interfacial region and (ii) changes in the driving force for the heterogeneous electron transfer. A comparison between the potential dependence of the photocurrent and capacitance values in the presence of TCNQ suggests that voltage induced changes in the particle coverage do affect the photocurrent conversion efficiency. Indeed, the onset of the photocurrent responses is close to $0 \mathrm{~V}$, which coincides with the onset potential for the adsorption of CdSe particles at the interface.

The photocurrent in the presence of TCNQ, tetrachloro-1,4-benzoquinone (TCBQ) and 2,6-dichloro-1,4benzoquinone (DCBQ) at the same Galvani potential difference is contrasted in Fig. 6. These results clearly show that the photocurrent decreases as the redox potential of the acceptor is more negative. In the case of DCBQ, the photocurrent signal is significant at potentials more negative than $-0.3 \mathrm{~V}$, i.e. at the edge of the polarisable window. In addition, no photocurrent

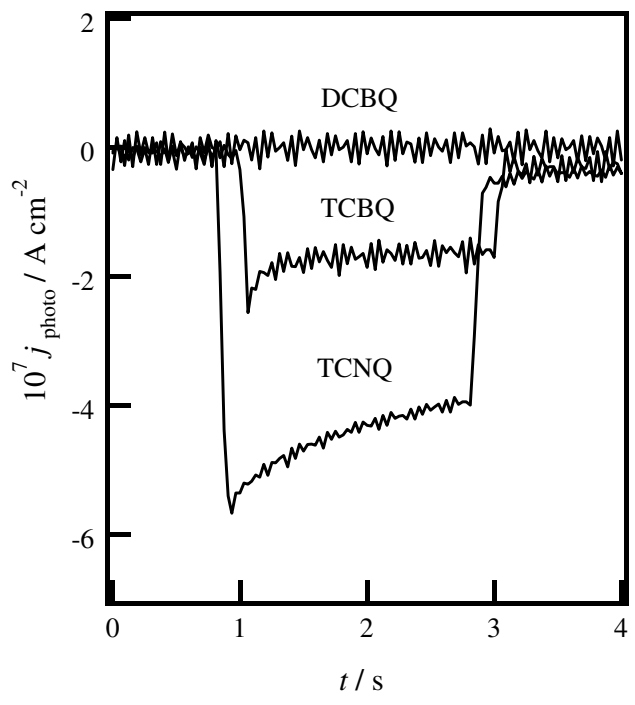

Fig. 6. Photocurrent transients at $-0.22 \mathrm{~V}$ in the presence of various electron acceptors. The concentration of the nanoparticles and electron acceptor were $0.25 \mathrm{~g} \mathrm{~L}^{-1}$ and $1 \times 10^{-3} \mathrm{M}$, respectively. The incident photon flux was $1.10 \times 10^{17} \mathrm{~cm}^{-2} \mathrm{~s}^{-1}$.

responses were observed in the presence of benzoquinone (BQ), which has a formal redox potential of $-0.34 \mathrm{~V}$ vs. SHE in bulk DCE. The dependence of the photocurrent on the redox potential of the electron acceptor indicates that the photoinduced electron transfer reaction is also kinetically controlled.

A strategy to uncouple the potential dependences of the particle interfacial density and electron transfer rate involves the study of the photocurrent responses associated with the transfer of photogenerated holes. For instance, the redox potentials of ferrocene and decamethylferrocene are 0.64 and $0.02 \mathrm{~V}$ vs. SHE, respectively, which are well above the valence band of the CdSe nanoparticle and enable them to act as effective scavengers for photogenerated holes. However, no photocurrents were observed in the presence of the electron donors in DCE over the whole potential range. These results indicate that the photogenerated holes are effectively quenched by a fast homogeneous process.

\subsection{Dynamic aspects of the photoinduced responses}

The results illustrated in Figs. 5 and 6 clearly show that photoinduced heterogeneous electron transfer only occurs to redox species featuring potential significantly more positive than $E_{\mathrm{CB}}$ Considering that the electron transfer most probably takes place in the microsecond time scale due to the weak electronic coupling between donors and acceptors [10], we suggest that the electron injection occurs via deep trap states at the particle surface. Trapped charge carriers in localised surface states can exhibit remarkably slow depopulation time constants (>1 ms) [39]. Indeed, the red-shifted and low yield luminescence observed for these quantum dots indicates 
that most of the photogenerated carriers are trapped by interband states. The dynamics of population/depopulation of trap states are beyond the scope of this paper. In the subsequent discussion, we shall describe the overall relaxation of photogenerated electrons by a phenomenological rate constant $k_{\mathrm{d}}\left(\mathrm{s}^{-1}\right)$. This parameter represents the electron trapping rate constant integrated over the energy range of depopulated states in the band gap.

Previous models developed for dye sensitised liquid| liquid interfaces describe the photocurrent responses as a result of a series of competing reactions $[6,7,10,11]$. The first competition upon illumination involves the relaxation of the photogenerated electron $\left(k_{\mathrm{d}}\right)$ and the photoinduced heterogeneous electron transfer. These processes are followed by the competition between the back electron transfer reaction $\left(k_{\mathrm{b}}\right)$ regenerating the initial reactants and the product separation process $\left(k_{\mathrm{ps}}\right)$ leading to the final products in their respective electrolyte phases. Assuming that the interfacial concentration of the particles is time independent, the photocurrent responses can be expressed in the Laplace plane as [40]

$\overline{\Delta j_{\mathrm{ph}}}=\frac{F g}{1+R C_{\mathrm{dl} l} s} \frac{k_{\mathrm{ps}}+s}{\left(s+k_{\mathrm{b}}+k_{\mathrm{ps}}\right)+\left(s+k_{\mathrm{ps}}\right) \lambda_{\mathrm{et}} s^{1 / 2}}$,

where

$g=\frac{k_{\mathrm{et}}^{\mathrm{II}} c_{\mathrm{Q}}^{\mathrm{o}}}{k_{\mathrm{et}}^{\mathrm{II}} c_{\mathrm{Q}}^{\mathrm{o}}+k_{\mathrm{d}}} \sigma I_{0} \Gamma$

$\lambda_{\mathrm{et}}=\frac{g}{\left(D_{\mathrm{Q}}^{\mathrm{o}}\right)^{1 / 2} c_{\mathrm{Q}}^{\mathrm{o}}}$.

$k_{\text {et }}^{\mathrm{II}}$ is the second-order rate constant with respect to the populated density states in the particle and unoccupied density states in the redox species, $c_{\mathrm{Q}}^{\mathrm{o}}$ is the initial concentration of electron acceptors, $I_{0}$ is the photon flux and $\Gamma$ the interfacial concentration of particle at the liquid|liquid boundary. The second term in Eq. (3) not only describes the competition between product separation and back electron transfer, but also the diffusion of the acceptor to the interface upon continuous illumination (represented by the diffusion coefficient $D_{\mathrm{Q}}^{\mathrm{o}}$ ). The parameter $R C_{\mathrm{dl}}$ relates to the attenuation of the photoresponses at short time determined by the uncompensated resistance $R$ and the double layer capacitance $C_{\mathrm{dl}}$.

The dotted lines in Fig. 5 correspond to numerical solutions of Eqs. (3)-(5) employing $g, k_{\mathrm{b}}$ and $k_{\mathrm{ps}}$ as adjustable parameters. $D_{\mathrm{Q}}^{\mathrm{o}}$ was set to $1.05 \times$ $10^{-5} \mathrm{~cm}^{-2} \mathrm{~s}^{-1}$ according to previous reports $[41,42]$, and $R C_{\mathrm{dl}}$ was estimated as $0.025 \mathrm{~s}^{-1}$ from the admittance measurements. The rising portion of the photocurrent is determined by the $R C_{\mathrm{dl}}$ component, while the response decay is sensitive to $D_{\mathrm{Q}}^{\mathrm{o}}$ and the rate constants $k_{\mathrm{b}}$ and $k_{\mathrm{ps}}$. On the other hand, the initial magnitude of the photocurrent is determined by the parameter $g$ (Eq. (4)). As illustrated in Fig. 5, consistent fits were obtained for values of $g$ of $2.30 \times$
$10^{-10} \mathrm{~mol} \mathrm{~cm}^{-2} \mathrm{~s}^{-1}$ at $-0.12 \mathrm{~V}, 4.80 \times 10^{-10} \mathrm{~mol} \mathrm{~cm}^{-2} \mathrm{~s}^{-1}$ at $-0.17 \mathrm{~V}$ and $6.30 \times 10^{-10} \mathrm{~mol} \mathrm{~cm}^{-2} \mathrm{~s}^{-1}$ at $-0.22 \mathrm{~V}$. As discussed in the previous section, the dependence of $g$ on the Galvani potential difference is determined by not only changes in $\Gamma$ but also the rate of electron transfer $k_{\mathrm{et}}^{\mathrm{II}}$. Independent analysis of the interfacial concentration of the particles as a function of the applied potential would be required to further rationalise these results. Taking into account the values obtained at $-0.22 \mathrm{~V}$ in the presence of TCNQ, the photocurrent conversion efficiency given by the ratio between $g$ and the photon flux $I_{0}$ can be estimated as $0.35 \%$.

The analysis of the photocurrent decay shows that the most important contribution arises from the diffusion of the electron acceptor to the interfacial region. However, at high photocurrent densities it appears that back electron transfer also contributes to the decay of the photocurrent. This behaviour may arise from the fact that the model considers the back electron transfer as a first order reaction with respect to intermediate charge transfer complex formed after the heterogeneous electron transfer step [40]. Although this approximation is validated by experimental results in the case of porphyrin sensitised liquid|liquid interfaces, it may not be applicable to the CdSe system. However, the key result is that the photocurrent decay is mostly determined by the diffusion of the acceptor in the organic phase. In phenomenological terms, the photoelectrochemical responses resemble those observed for p-type semiconductor in the depletion potential range. Obviously, there is no such depletion layer in the CdSe nanoparticles inducing the migration of majority carriers. In this case, the fast quenching of holes by species in the aqueous phase is the physical phenomena behind the depletion of holes at the liquid| liquid boundary.

\section{Conclusions}

Water-soluble MSA protected CdSe nanoparticles can be reversibly adsorbed at the water|DCE interface by tuning the Galvani potential difference. In the potential range where the nanoparticles are adsorbed, band-gap illumination leads to heterogeneous electron transfer from CdSe nanoparticles to electron scavengers located in the organic phase. On the other hand, no photocurrents were observed in the presence of electron donors, indicating that valence band holes are swiftly removed. These facts indicate that only photoexcited electrons are kinetically allowed to be transferred heterogeneously across the liquid|liquid interface.

The photocurrent efficiency strongly depends on the applied Galvani potential difference and the formal redox potential of the electron acceptor. These results not only reflect the role of the Galvani potential differ- 
ence on the number density of nanoparticles at the interface, but also on the dynamics of heterogeneous electron transfer. It is concluded that trapped electrons are involved as intermediates in the heterogeneous photoreaction. Analysis of the photocurrent transients reveals that the initial photocurrent magnitude is determined by the competition between electron relaxation and heterogeneous electron transfer, while the photocurrent decay upon constant illumination is mostly determined by the diffusion of the acceptor to the interfacial region.

\section{Acknowledgements}

This work was supported by the Fonds National Suisse de la Recherche Scientifique (Project 2000067050.01). We are grateful to Prof. Philippe Buffat for HRTEM measurements and Valérie Devaud for technical assistance.

\section{References}

[1] F. Reymond, D. Fermin, H.J. Lee, H.H. Girault, Electrochim. Acta 45 (2000) 2647.

[2] D.J. Fermin, R. Lahtinen, Surf. Sci. Ser. 95 (2001) 179.

[3] R. Lahtinen, H. Jensen, D.J. Fermin, Interf. Catal. (2003) 611.

[4] D.J. Fermin, Z. Ding, H.D. Duong, P.-F. Brevet, H.H. Girault, J. Phys. Chem. B 102 (1998) 10334.

[5] D.J. Fermin, H.D. Duong, Z. Ding, P.F. Brevet, H.H. Girault, Electrochem. Commun. 1 (1999) 29.

[6] D.J. Fermin, H. Dung Duong, Z. Ding, P.F. Brevet, H.H. Girault, Phys. Chem. Chem. Phys. 1 (1999) 1461.

[7] D.J. Fermin, H.D. Duong, Z. Ding, P.-F. Brevet, H.H. Girault, J. Am. Chem. Soc. 121 (1999) 10203.

[8] H. Jensen, J.J. Kakkassery, H. Nagatani, D.J. Fermin, H.H Girault, J. Am. Chem. Soc. 122 (2000) 10943.

[9] H. Jensen, D.J. Fermin, H.H. Girault, Phys. Chem. Chem. Phys. 3 (2001) 2503.

[10] N. Eugster, D.J. Fermin, H.H. Girault, J. Phys. Chem. B 106 (2002) 3428.

[11] N. Eugster, D.J. Fermin, H.H. Girault, J. Am. Chem. Soc. 125 (2003) 4862.

[12] N. Eugster, H. Jensen, D.J. Fermin, H.H. Girault, J. Electroanal. Chem. 560 (2003) 143.

[13] D.J. Fermin, H. Jensen, J.E. Moser, H.H. Girault, Chem. Phys. Chem. 4 (2003) 85.

[14] H. Jensen, D.J. Fermin, J.E. Moser, H.H. Girault, J. Phys. Chem. B 106 (2002) 10908.
[15] E.P.A.M. Bakkers, A.W. Marsman, L.W. Jenneskens, D. Vanmaekelbergh, Angew. Chem., Int. Ed. 39 (2000) 2297.

[16] E.P.A.M. Bakkers, A.L. Roest, A.W. Marsman, L.W. Jenneskens, L.I. De Jong-Van Steensel, J.J. Kelly, D. Vanmaekelbergh, J. Phys. Chem. B 104 (2000) 7266.

[17] M. Miyake, T. Torimoto, M. Nishizawa, T. Sakata, H. Mori, H. Yoneyama, Langmuir 15 (1999) 2714.

[18] A.P. Alivisatos, Science 271 (1996) 933.

[19] M. Nirmal, L. Brus, Accounts Chem. Res. 32 (1999) 407.

[20] J.Z. Zhang, J. Phys. Chem. B 104 (2000) 7239.

[21] E.R. Goldman, G.P. Anderson, P.T. Tran, H. Mattoussi, P.T. Charles, J.M. Mauro, Anal. Chem. 74 (2002) 841.

[22] H. Mattoussi, J.M. Mauro, E.R. Goldman, G.P. Anderson, V.C. Sundar, F.V. Mikulec, M.G. Bawendi, J. Am. Chem. Soc. 122 (2000) 12142.

[23] S. Coe, W.-K. Woo, M. Bawendi, V. Bulovic, Nature 420 (2002) 800.

[24] V.L. Colvin, M.C. Schlamp, A.P. Alivisatos, Nature 370 (1994) 354.

[25] M.C. Schlamp, X. Peng, A.P. Alivisatos, J. Appl. Phys. 82 (1997) 5837.

[26] C. Wang, M. Shim, P. Guyot-Sionnest, Science 291 (2001) 2390.

[27] R. Plass, S. Pelet, J. Krueger, M. Graetzel, U. Bach, J. Phys. Chem. B 106 (2002) 7578

[28] M. Bruchez Jr., M. Moronne, P. Gin, S. Weiss, A.P. Alivisatos, Science 281 (1998) 2013

[29] W.C. Chan, S. Nie, Science 281 (1998) 2016.

[30] E.R. Goldman, E.D. Balighian, H. Mattoussi, M.K. Kuno, J.M. Mauro, P.T. Tran, G.P. Anderson, J. Am. Chem. Soc. 124 (2002) 6378.

[31] B.M. Lingerfelt, H. Mattoussi, E.R. Goldman, J.M. Mauro, G.P. Anderson, Anal. Chem. 75 (2003) 4043.

[32] A.L. Rogach, A. Kornowski, M. Gao, A. Eychmueller, H. Weller, J. Phys. Chem. B 103 (1999) 3065.

[33] B. Su, J.-P. Abid, D.J. Fermin, H.H. Girault, H. Hoffmannova, P. Krtil, Z. Samec, J. Am. Chem. Soc. 126 (2004) 915.

[34] S. Chen, K. Kimura, Langmuir 15 (1999) 1075.

[35] M. Gao, B. Richter, S. Kirstein, H. Moehwald, J. Phys. Chem. B 102 (1998) 4096.

[36] C.A. Leatherdale, W.K. Woo, F.V. Mikulec, M.G. Bawendi, J. Phys. Chem. B 106 (2002) 7619.

[37] E.D. Palik, Handbook of Optical Constants II, vol. 2, Academic Press, Boston, 1991.

[38] C. Burda, T.C. Green, S. Link, M.A. El-Sayed, J. Phys. Chem. B 103 (1999) 1783.

[39] M. Shim, S.V. Shilov, M.S. Braiman, P. Guyot-Sionnest, J. Phys. Chem. B 104 (2000) 1494.

[40] Z. Samec, N. Eugster, D.J. Fermin, H.H. Girault, J. Electroanal. Chem. 577 (2005) 323.

[41] Z. Ding, D.J. Fermin, P.-F. Brevet, H.H. Girault, J. Electroanal. Chem. 458 (1998) 139.

[42] J. Zhang, P.R. Unwin, Phys. Chem. Chem. Phys. 4 (2002) 3820. 